\title{
Analysis of stationary processes using fuzzy transform
}

\author{
Michal Holčapek Vilém Novák Irina Perfilieva
}

CE IT4Innovations - Division of the University of Ostrava - IRAFM, 30. dubna 22, 70103 Ostrava, CZ

\begin{abstract}
In this paper, we show that fuzzy transform originally introduced for a transformation of complex spaces of functions to simpler ones can be used in the analysis of real stationary random processes. We will show that under certain assumptions the fuzzy transform may be used for an approximation of this type of stationary processes as well as for a reduction of their variability. The obtained results could help researches to understand better the analysis of time series based on fuzzy transform.
\end{abstract}

Keywords: Fuzzy transform, uniform fuzzy partition, stationary random process

\section{Introduction}

In time series analysis, random noise component is assumed in many cases to be a stationary process for its valuable properties. Fuzzy transform (F-transform for short) is a technique based on a partitioning of a real interval using fuzzy sets that generally transforms complex spaces of functions to simpler ones. By setting of fuzzy partition parameters the F-transform can be used for approximation and smoothing of original functions. The latter has been used among others in time series analysis for a trend extraction and a reduction of seasonal components (see [1], [2]).

In this paper, we focus on the random noise component of time series described by a weakly stationary process (with zero mean value). We will show that under specific assumptions the F-transform can be used for an approximation of this type of stationary process as well as for a reduction of variability.

The first result is motivated by the idea to represent complex stationary processes using processes with a discrete spectrum. It can be shown (see, e.g., [3]) that each stationary process $\xi(t)$ defined on a wide interval $[-T, T]$ (it means for a large $T$ ) can be approximated arbitrarily closely by a linear combination of harmonic oscillations of the form

$$
\sum_{j=1}^{n} \xi_{j} e^{i \lambda_{j} t}
$$

where $\xi_{1}, \ldots, \xi_{n}$ are pairwise uncorrelated random variables with mean zero independent on time $t, i$ is the imaginary unit and $\lambda_{1}, \ldots, \lambda_{n}$ are real constant. More precisely, it can be proved that for any $\varepsilon>0$ there exist random variables $\xi_{1}, \ldots, \xi_{n}$ which are pairwise uncorrelated and real numbers $\lambda_{1}, \ldots, \lambda_{n}$ such that

$$
\mathbf{E}\left[\xi(t)-\sum_{j=1}^{n} \xi_{j} e^{i \lambda_{j} t}\right]<\varepsilon
$$

for any $t \in[-T, T]$. The representation of real stationary processes by F-transform, however, keeps a different idea than the previous one. The linear combination of harmonic oscillations is here replaced by the combination of basic functions which uniformly partition the real line and the closeness of frequencies $\lambda_{j}$ by closeness of nodes over which fuzzy partitions are built.

The presented results have to be considered as preliminary ones justifying the investigation of stationary processes using F-transform. Another argument supporting the investigation of F-transform in the area of stationary processes comes from the computation complexity $O(n)$ of F-transform in contrast to the computational complexity $O(n \log n)$ that holds for the fast Fourier transform.

The second result of this paper is motivated by a lack of proper theoretical justification of the fact that the F-transform can be applied in time series analysis for filtering out a random noise.

The paper is structured as follows. A necessary background for the analysis of stationary processes including two proofs of integral inequalities (in the mean) for them is provided in next section. The third section is devoted to the basic F-transform concepts translated into the language of stochastic processes. The main results are presented in the fourth and fifth section. The last section is a conclusion.

\section{Stationary processes}

In this section, we provide a necessary background for our analysis of weakly stationary process $\xi(t)$ by fuzzy transform.

\subsection{Assumptions}

In what follows, we assume that a probability space $(\Omega, \mathcal{F}, P)$ is fixed and we consider a real random process $\xi(t)$ (defined for any real number $t$ ) such that for any finite sequence $t_{1}, \ldots, t_{n}(n=1,2, \ldots)$ 
of times there is a joint distribution function given by

$$
\begin{aligned}
& F_{t_{1}, \ldots, t_{n}}\left(x_{1}, \ldots, x_{n}\right)= \\
& P\left(\left\{\xi\left(t_{1}\right) \leq x_{1}, \ldots, \xi\left(t_{n}\right) \leq x_{n}\right\}\right) .
\end{aligned}
$$

The distribution functions (1) must satisfy the following two conditions:

(D1) The symmetry condition, according to which $F_{t_{i_{1}}, \ldots, t_{i_{n}}}\left(x_{i_{1}}, \ldots, x_{i_{n}}\right)=F_{t_{1}, \ldots, t_{n}}\left(x_{1}, \ldots, x_{n}\right)$, where $i_{1}, \ldots, i_{n}$ is a permutation of the indices $1, \ldots, n$;

(D2) The compatibility condition, according to which

$$
\begin{gathered}
F_{t_{1}, \ldots, t_{m}, t_{m+1}, \ldots, t_{n}}\left(x_{1}, \ldots, x_{m}, \infty, \ldots, \infty\right)= \\
F_{t_{1}, \ldots, t_{m}}\left(x_{1}, \ldots, x_{m}\right)
\end{gathered}
$$

for any $t_{m+1}, \ldots, t_{n}$ if $m<n$.

We use E, Var, Cov to denote the expected value, variance and covariance of random variables. Further, let us define a real function $B(t, s)$ called covariance function of $\xi(t)$ (see [4] ) by

$$
B(t, s)=\mathbf{E}[\xi(t) \xi(s)] .
$$

We assume that $\xi(t)$ satisfies the following conditions for any $t$ :

(i) $\mathbf{E}[\xi(t)]=0$;

(ii) $B(t, t+\tau)$ is independent of $t$ for each $\tau$;

(iii) $B(\tau)=B(0, \tau)$ is Lebesgue integrable.

The first two conditions says that $\xi(t)$ is a (weakly) stationary process, the latter is a necessary condition for our analysis. Obviously, the covariance of random variables $\xi(t)$ and $x i(s)$ is equal to $B(t-s)$, i.e.,

$$
\operatorname{Cov}(\xi(t), \xi(s))=B(t-s) .
$$

Specifically, we have $\operatorname{Var}(\xi(t))=B(0)=\sigma^{2}$. Then,

$$
|B(\tau)| \leq B(0)=\sigma^{2}
$$

for any $t, s .^{1}$ Note that the previous inequality says that the random variables $\xi(t)$ and $\xi(s)$ are dependent to each other in a degree which absolute value is at most equal to the variance of $\xi(t)$. Sometimes, it seems to be natural to assume that higher difference between $t$ and $s$ causes lower dependence, i.e., $B(\tau)$ is (continuously) going down to 0 for $|\tau|$ is going up to greater numbers. The assumption on integrability of $B(\tau)$ is a necessary condition for our analysis of approximation and variability reduction of $\xi(t)$ using the fuzzy transform.

\footnotetext{
${ }^{1}$ It follows from the Cauchy-Schwartz's inequality.
}

\subsection{Limit of sequences of random variables}

Let $\xi_{1}, \xi_{2}, \ldots$ be a sequence of random variables. We say that a random variable $\xi$ is a limit in the mean square of the sequence of random variables $\xi_{1}, \xi_{2}, \ldots$ and denote it by

$$
\lim _{n \rightarrow \infty} \xi_{n}=\xi
$$

if

$$
\lim _{n \rightarrow \infty} \mathbf{E}\left[\left(\xi_{n}-\xi\right)^{2}\right]=0,
$$

i.e., for any $\varepsilon>0$ there exists a natural number $n_{0}$ such that

$$
\mathbf{E}\left[\left(\xi_{n}-\xi\right)^{2}\right]<\varepsilon
$$

for any $n>n_{0} \cdot{ }^{2}$ Let us show two important properties of limit in the mean square which will be used later (see [5]).

Theorem 1 Let $\left(\xi_{n}\right)_{n=1}^{\infty}, \quad\left(\psi_{n}\right)_{n=1}^{\infty}$ be two sequences of random variables and let us suppose that l.i. $\mathrm{m}_{n \rightarrow \infty} \xi_{n}=\xi$ and l. i. $\mathrm{m}_{n \rightarrow \infty} \psi_{n}=\psi$. Then,

(i) $\mathbf{E}[\xi]=\lim _{n \rightarrow \infty} \mathbf{E}\left[\xi_{n}\right]$,

(ii) $\mathbf{E}[\xi \psi]=\lim _{n \rightarrow \infty} \mathbf{E}\left[\xi_{n} \psi_{n}\right]$.

\subsection{Integral of stationary process}

Let $f(t)$ be an arbitrary real function and $\xi(t)$ a stationary random process. The integral

$$
\int_{c}^{d} \xi(t) f(t) d t
$$

is defined as the limit (in the mean square) of random variables

$$
\sum_{j=2}^{n} \xi\left(t_{j}^{\prime}\right) f\left(t_{j}^{\prime}\right)\left(t_{j}-t_{j-1}\right),
$$

where $c=t_{1}<t_{2}<\cdots<t_{n}=d$ and $t_{j-1} \leq t_{j}^{\prime} \leq t_{j}$ holds for any $j=2, \ldots, n$. Of course, this integral does not exist for all pairs of real functions and stationary processes. For details, we refer to [3]. In what follows, we will assume only such real functions and stationary processes that are integrable with respect to the integral (7).

In order to show the approximation of stationary processes using the F-transform, we will need the following special case of Hölder's inequality for integrals which holds in the mean.

Theorem 2 Let $\xi(t)$ be a stationary process and $f(t)$ be a real function defined on $[c, d]$. Then,

$$
\begin{gathered}
\mathbf{E}\left[\left(\int_{c}^{d}|\xi(t) f(t)| d t\right)^{2}\right] \leq \\
\mathbf{E}\left[\int_{c}^{d}|\xi(t)|^{2} d t \int_{c}^{d}|f(t)|^{2} d t\right]
\end{gathered}
$$

\footnotetext{
${ }^{2}$ Note that we use the symbol l.i.m to distinguish the limit in the mean square of a sequence of random variables and the common limit of a sequence of numbers (cf., [5]).
} 
Proof: The following inequality is a special form of Hölder's inequality used in the probability theory

$$
\mathbf{E}\left[\left|\xi_{1} \xi_{2}\right|\right] \leq\left(\mathbf{E}\left[\left|\xi_{1}\right|^{2}\right]^{\frac{1}{2}}\left(\mathbf{E}\left[\left|\xi_{2}\right|^{2}\right]\right)^{\frac{1}{2}}\right.
$$

Using (ii) of Theorem 1 and the definition of integral (7), we obtain

$$
\begin{gathered}
\mathbf{E}\left[\left(\int_{c}^{d}|\xi(t) f(t)| d t\right)^{2}\right]= \\
\lim _{n \rightarrow \infty} \mathbf{E}\left[\left(\sum_{j=2}^{n}\left|\xi\left(t_{j}^{\prime}\right) f\left(t_{j}^{\prime}\right)\right|\left(t_{j}-t_{j-1}\right)\right)^{2}\right]= \\
\lim _{n \rightarrow \infty} \sum_{i=2}^{n} \sum_{j=2}^{n} \mathbf{E}\left[\left|\xi\left(t_{i}^{\prime}\right) \xi\left(t_{j}^{\prime}\right)\right|\right]\left|f\left(t_{i}^{\prime}\right) f\left(t_{j}^{\prime}\right)\right| \cdot \\
\cdot\left(t_{i}-t_{i-1}\right)\left(t_{j}-t_{j-1}\right) \leq \\
\lim _{n \rightarrow \infty} \sum_{i=2}^{n} \sum_{j=2}^{n}\left(\mathbf{E}\left[\left|\xi\left(t_{i}^{\prime}\right)\right|^{2}\right]^{\frac{1}{2}}\left(\mathbf{E}\left[\left|\xi\left(t_{j}^{\prime}\right)\right|^{2}\right]\right)^{\frac{1}{2}}\left|f\left(t_{i}^{\prime}\right) f\left(t_{j}^{\prime}\right)\right| \cdot\right. \\
\cdot\left(t_{i}-t_{i-1}\right)\left(t_{j}-t_{j-1}\right)= \\
\lim _{n \rightarrow \infty}\left(\sum_{i=2}^{n}\left(\mathbf{E}\left[\left|\xi\left(t_{i}^{\prime}\right)\right|^{2}\right]^{\frac{1}{2}}\left|f\left(t_{i}^{\prime}\right)\right|\left(t_{i}-t_{i-1}\right)\right)^{2}=\right. \\
\left(\lim _{n \rightarrow \infty} \sum_{i=2}^{n} \mathbf{E}\left[\left|\xi\left(t_{i}^{\prime}\right)\right|^{2}\right]^{\frac{1}{2}}\left|f\left(t_{i}^{\prime}\right)\right|\left(t_{i}-t_{i-1}\right)\right)^{2}= \\
\left(\int_{c}^{d} \mathbf{E}\left[|\xi(t)|^{2}\right]^{\frac{1}{2}}|f(t)| d t\right)^{2} \cdot
\end{gathered}
$$

By the Hölder's inequality for integrals, we obtain

$$
\begin{gathered}
\left(\int_{c}^{d} \mathbf{E}\left[|\xi(t)|^{2}\right]^{\frac{1}{2}}|f(t)| d t\right)^{2} \leq \\
\left(\left(\int_{c}^{d} \mathbf{E}\left[|\xi(t)|^{2}\right] d t\right)^{\frac{1}{2}}\left(\int_{c}^{d}|f(t)|^{2} d t\right)^{\frac{1}{2}}\right)^{2}
\end{gathered}
$$

which implies the Hölder's type of inequality in the mean for integrals of stationary processes.

Since $\mathbf{E}[\xi(t) f(t)] \leq \mathbf{E}[|\xi(t) f(t)|]$, one can simply check the integral inequality for absolute value which holds in the mean.

Theorem 3 Let $\xi(t)$ be a stationary random process and $f(t)$ be a real function defined on $[c, d]$. Then,

$$
\mathbf{E}\left[\int_{c}^{d} \xi(t) f(t) d t\right] \leq \mathbf{E}\left[\int_{c}^{d}|\xi(t) f(t)| d t\right] .
$$

Proof: Obvious.

\section{F-transform of stationary process}

In this section, we will briefly review the main principles of the fuzzy transform. Detailed explanation of the general theory can be found in $[6,7,8]$.
Let $U$ be an arbitrary (nonempty) set called a universe. By a fuzzy set in the universe $U$ we will understand a function $A: U \rightarrow[0,1]$.

The F-transform is a special technique that can be applied to real continuous functions $f$, defined on an interval $[a, b] \subset \mathbb{R}$. The essential idea is to transfer $f$ into another, simpler space, and then to transfer the respective image back. The latter space consists of finite vectors that are obtained on the basis of the well formed fuzzy partitions of the domain of the given function. Thus, the first step called direct F-transform results in the vector of averaged functional values. The second step called inverse $F$ transform converts this vector into another continuous function $\hat{f}$, which approximately reconstructs the original $f$.

\subsection{Uniform fuzzy partition}

Let $\mathbb{Z}$ denote the set of integers. It is well-known that a uniform fuzzy partition is defined using a generating function $K$ which is modified by a parameter $h$ expressing the required spread. Each basic function of the uniform fuzzy partition is then constructed using a suitable shift of the modified generating function $K$, where the uniformity for all shifts is supposed. The generating function is defined as follows.

Definition 1 A function $K: \mathbb{R} \rightarrow[0,1]$ is said to be a generating function if $K$ is an even Lebesgue integrable function (fuzzy set) which is non-increasing in $[0, \infty)$ and

$$
K(x) \begin{cases}>0, & \text { if } x \in(-1,1) ; \\ =0, & \text { otherwise. }\end{cases}
$$

$A$ generating function $K$ is said to be normal if $K(0)=1$.

It should be noted that the previous definition is more general than the analogous definition of a generating function in [9], because the continuity of $K$ is replaced by its integrability and the normality of $K$ is considered as an additional condition. ${ }^{3}$ Uniform fuzzy partitions of the real line are defined as follows (cf., [11]).

Definition 2 Let $K$ be a normal generating function, $h$ be a positive real number and $c_{0} \in \mathbb{R}$. A system of fuzzy sets defined by

$$
A_{k}(x)=K\left(\frac{x-c_{0}}{h}-k\right)
$$

for any $k \in \mathbb{Z}$ is said to be a uniform fuzzy partition (UFP) of the real line determined by the triplet $\left(K, h, c_{0}\right)$ if the Ruspini's condition is satisfied, i.e.,

$$
S(x)=\sum_{k \in \mathbb{Z}} A_{k}(x)=1
$$

holds for any $x \in \mathbb{R}$.

\footnotetext{
${ }^{3}$ In [10], a generating function was called a basal function.
} 
In the sequel, the parameters $h$ and $c_{0}$ are called a spread and a central node, respectively. The fuzzy sets $A_{k}$ defined by (12) that form a uniform fuzzy partition of the real line are called basic functions. A simple consequence of (12) is the formula $A_{k}(x)=$ $A_{0}(x-h k)$ that holds for any $x \in \mathbb{R}$ and $k \in \mathbb{Z}$. Putting $c_{k}=c_{0}+k h$ one can simply check that $A_{k}\left(c_{k}\right)=1$ and $A_{k}$ is centered around the node $c_{k}$.

Remark 1 (Important) One can see that uniform fuzzy partitions of closed real intervals used in the fuzzy transform can be simply spread out to uniform fuzzy partitions of the real line. Therefore, each uniform fuzzy partitions of a closed real interval can be understood as a partition of the real line which is limited to the closed real interval, and we can restrict our investigation properties of uniform fuzzy partitions to the partitions of the real line. In the sequel for the sake of simplicity, we will omit "the real line" in "uniform fuzzy partition or UFP of the real line", and we will speak only about uniform fuzzy partitions or UFPs.

Let us show two most usable examples of generating function and a uniform fuzzy partition determined by this function (see [6]).

\section{Example 1 (Triangle generating function)}

Let $K: \mathbb{R} \rightarrow[0,1]$ be defined by

$$
K_{T}(x)=\max (1-|x|, 0) .
$$

On Figure 1, one can see a part of the UFP of $\mathbb{R}$ determined by $\left(K_{T}, 2,1\right)$. The dashed function displays the triangular generating function with the bandwidth $h=2$, and, for example, the basic function $A_{2}$ is obtained by shifting the center 0 of the generating function $K_{T}$ to the new center (node) $c_{2}=c_{0}+2 h=1+2 \cdot 2=5$.

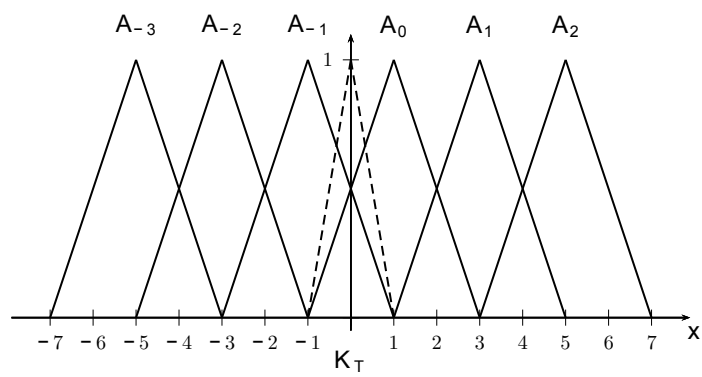

Figure 1: A part of the UFP of the real line determined by $\left(K_{T}, 2,1\right)$. The dashed function displays the triangle generating function $K_{T}$ which is centered around 0 .

Example 2 (Cosine generating function) Let $K: \mathbb{R} \rightarrow[0,1]$ be defined by

$$
K_{C}(x)= \begin{cases}\frac{1}{2}(1+\cos (\pi x)), & -1 \leq x \leq 1 \\ 0, & \text { otherwise }\end{cases}
$$

On Fig. 2, one can see a part of the UFP of $\mathbb{R}$ determined by $\left(K_{C}, 2,1\right)$. The dashed function displays the raised cosine generating function with the bandwidth $h=2$ and the central node $c_{0}=1$.

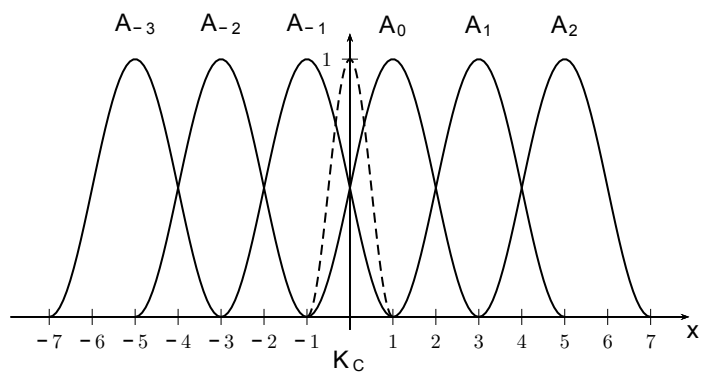

Figure 2: A part of the UFP of the real line determined by $\left(K_{C}, 2,1\right)$. The dashed function displays the raised cosine generating function $K_{C}$ which is centered around 0 .

\subsection{Direct and inverse F-transform}

We use $\mathbf{A}_{h}=\left(A_{k}\right)_{k \in \mathbb{Z}}$ to denote a uniform fuzzy partition of real line determined by $\left(K, h, x_{0}\right)$ and denote $\left(c_{k}\right)_{k \in \mathbb{Z}}$ their corresponding nodes, i.e., $A_{k}(x)=A_{0}\left(x-c_{k}\right)=K\left(\frac{x-c_{k}}{h}\right)$.

Remark 2 (Important) It should be stressed that we deal here with functions which domain is the real line. Similarly to Remark 1 we can extend a function $f$ defined on $[a, b]$ to be defined on $\mathbb{R}$ by putting $f(t)=0$ for any $t \notin[a, b]$. It is clear that the approximation of functions with finite domains by the F-transform provided in [9] can be equivalently done using F-transform defined over infinite uniform fuzzy partitions and functions with the infinite domain $(-\infty, \infty)$. Moreover, it seems that a problem with the basic functions which form the boundary of finite UFPs is automatically excluded in this case. However, it does not mean that a function extended from $[a, b]$ to the real line will have a better approximation around the boundaries $a$ and $b$ than in the case where a finite UFP is considered.

Definition 3 Let $\xi(t)$ be a stationary process, $\mathbf{A}_{h}$ be a uniform fuzzy partition and $\left(c_{k}\right)_{k \in \mathbb{Z}}$ denote the respective nodes. An infinite vector of random variables $\left(\xi_{k}\right)_{k \in \mathbb{Z}}$ is called a direct fuzzy transform (Ftransform) of $\xi(t)$ with respect to $\mathbf{A}_{h}$ if

$$
\xi_{k}=\frac{1}{h} \int_{c_{k-1}}^{c_{k+1}} \xi(t) A_{k}(t) d t, \quad k \in \mathbb{Z} .
$$

The random variable $\xi_{k}$ is called a component of F-transform.

It is easy to show that the linearity of F-transform is preserved for stationary processes, i.e., if $\xi(t)=$ $a \eta(t)+b \zeta(t), a, b \in \mathbb{R}$, then

$$
\xi_{k}=a \eta_{k}+b \zeta_{k} .
$$


Note that the linearity belongs among the most valuable properties of the F-transform often used in proofs.

In this paper, we use $\xi_{k, h}$ to denote the $k$-th Ftransform component at the node $c_{k}$ with respect to $\mathbf{A}_{h}$ and suppose only such stationary processes $\xi(t)$ for which $\xi_{k, h}$ can be found for any $k \in \mathbb{Z}$ and $h>0$.

The inverse $F$-transform is defined as the linear combination of components and basic functions. We use a slight modification of the original definition in [9] as follows.

Definition 4 Let $\xi(t)$ be a stationary process and $\left(\xi_{k}\right)_{k \in \mathbb{Z}}$ be the direct $F$-transform of $\xi(t)$ with respect to $\mathbf{A}_{h}$. Then,

$$
\hat{\xi}(t)=\sum_{k \in \mathbb{Z}} \xi_{k} A_{k}(t)
$$

is called an inverse $F$-transform of $\xi(t)$ with respect to $\mathbf{A}_{h}$.

The linearity of $F$-transform components (16) is preserved by the inverse $F$-transform as the following lemma shows.

Lemma 4 Let $\eta(t), \zeta(t), a, b \in \mathbb{R}$ and put $\xi(t)=$ $a \eta(t)+b \zeta(t)$. Then,

$$
\hat{\xi}(t)=a \hat{\eta}(t)+b \hat{\zeta}(t)
$$

Proof: Obvious.

\section{Approximation of $\xi(t)$ by $\mathbf{F}$-transform}

In order to investigate the approximation of stationary processes $\xi(t)$ by the F-transform, let $B^{*}(\tau)=$ $B(0)-B(\tau)$ and suppose that

$$
\lim _{h \rightarrow 0} \frac{1}{h} \int_{0}^{h} B^{*}(\tau) d \tau=0 .
$$

From (4), it is easy to see that the function $B^{*}(\tau)$ is a non-negative real function and the assumption (18) on $\xi(t)$ says that random variables $\xi(t)$ and $\xi(s)$ are very strongly dependent for small differences between $t$ and $s$, in other words, $B(\tau)$ converges to $B(0)$ for $\tau \rightarrow 0 .^{4}$

Example 3 (see Example 2, p. 33 in [3]) Let us consider a stationary process

$$
\xi(t)=\eta \cos \lambda t+\zeta \sin \lambda t,
$$

where $\eta$ and $\zeta$ are real random variables with $\mathbf{E}[\eta]=$ $\mathbf{E}[\zeta]=0, \operatorname{Var}[\eta]=\operatorname{Var}[\zeta]=b$ and $\operatorname{Cov}[\eta, \zeta]=0$. It is easy to verify that

$$
B(\tau)=b \cos \lambda \tau .
$$

\footnotetext{
${ }^{4}$ The latter follows from the integral mean value theorem saying that, for each $h>0$, there is $\tau^{\prime} \in(0, h)$ such that $1 / h \int_{0}^{h} B^{*}(\tau) d \tau=B^{*}\left(\tau^{\prime}\right)$, i.e., $B^{*}(\tau)$ converges to 0 .
}

Then, we obtain by simple computation that

$$
\lim _{h \rightarrow 0} \frac{1}{h} \int_{0}^{h} b(1-\cos \tau) d \tau=\lim _{h \rightarrow 0} b-\frac{b \sin \lambda h}{\lambda h}=0
$$

and $\xi(t)$ satisfies (18).

In what follows, we provide several theorems demonstrating how the F-transform can approximate stationary process satisfying (18). In the original paper ([6]) on the F-transform, the author shows that a twice continuously differentiable function differs from the F-transform components at nodes $c_{k}$ up to $h^{2}$. As a consequence we obtain that the F-transform components converge to the values of original function at nodes $c_{k}$ for $h \rightarrow 0$. The following theorem shows an analogous property for stationary stochastic process under the assumption (18).

Theorem 5 Let $\xi(t)$ satisfy (18) and $c_{k}$ be a fixed node. Then, there exists a sequence of F-transform components $\xi_{k, h_{1}}, \xi_{k, h_{2}}, \ldots$ at the node $c_{k}$ w.r.t. $\mathbf{A}_{h_{1}}, \mathbf{A}_{h_{2}}, \ldots$, respectively, such that

$$
\lim _{n \rightarrow \infty} \xi_{k, h_{n}}=\xi\left(c_{k}\right) .
$$

Proof: Let $h_{1}>h_{2}>\cdots>0$ be a sequence such that $\lim _{n \rightarrow \infty} h_{n}=0$ and define $A_{h_{n}}=\left(K, h_{n}, c_{k}\right)$. By the definition of $\xi_{k, h_{n}}$, one can simply check that (20) holds if and only if

$$
\lim _{n \rightarrow \infty} \frac{1}{h_{n}^{2}} \mathbf{E}\left[\left(\int_{c_{k-1}}^{c_{k+1}}\left(\xi(t)-\xi\left(c_{k}\right)\right) A_{k}(t) d t\right)^{2}\right]=0 .
$$

Put $\alpha_{h_{n}}=\int_{c_{k-1}}^{c_{k+1}}\left(A_{k}(t)\right)^{2} d t$. Since $c_{k+1}-c_{k-1}=$ $2 h_{n}, \alpha_{h_{n}} \leq 2 h_{n}$. Using the Hölder type of inequality (8) and (10), we obtain

$$
\begin{gathered}
\mathbf{E}\left[\left(\int_{c_{k-1}}^{c_{k+1}}\left(\xi(t)-\xi\left(c_{k}\right)\right) A_{k}(t) d t\right)^{2}\right] \leq \\
\mathbf{E}\left[\int_{c_{k-1}}^{c_{k+1}}\left(\xi(t)-\xi\left(c_{k}\right)\right)^{2} d t \int_{c_{k-1}}^{c_{k+1}}\left(A_{k}(t)\right)^{2} d t\right]= \\
\alpha_{h_{n}} \int_{c_{k-1}}^{c_{k+1}} \mathbf{E}\left[(\xi(t))^{2}-2 \xi(t) \xi\left(c_{k}\right)+\left(\xi\left(c_{k}\right)\right)^{2}\right] d t= \\
2 \alpha_{h_{n}} \int_{c_{k-1}}^{c_{k+1}}\left(B(0)-B\left(t-c_{k}\right)\right) d t= \\
2 \alpha_{h_{n}} \int_{-h_{n}}^{h_{n}} B^{*}(\tau) d \tau \leq 4 h_{n} \int_{-h_{n}}^{h_{n}} B^{*}(\tau) d \tau .
\end{gathered}
$$

By the assumption (18) and $\lim _{n \rightarrow \infty} h_{n}=0$, we simply obtain $\lim _{n \rightarrow \infty} \frac{4}{h_{n}} \int_{-h_{n}}^{h_{n}} B^{*}(\tau) d \tau=0$, hence

$$
\begin{gathered}
0 \leq \lim _{n \rightarrow \infty} \frac{1}{h_{n}^{2}} \mathbf{E}\left[\left(\int_{c_{k-1}}^{c_{k+1}}\left(\xi(t)-\xi\left(c_{k}\right)\right) A_{k}(t) d t\right)^{2}\right] \\
\leq \lim _{n \rightarrow \infty} \frac{4}{h_{n}} \int_{-h_{n}}^{h_{n}} B^{*}(\tau) d \tau=0
\end{gathered}
$$


and the proof is finished.

The following two theorems show that each stationary process $\xi(t)$ can be approximate arbitrarily closely by the F-transformed components belonging to the nearest neighborhood of $t$ (i.e., $[t-h, t+h]$ ).

Theorem 6 Let $\xi(t)$ satisfy (18) and $\left(\mathbf{A}_{h_{n}}\right)_{n=1}^{\infty}$ be a sequence of UFPs such that $\lim _{n \rightarrow \infty} h_{n}=0$. Then, for any $\varepsilon>0$, there exists $n_{0} \in \mathbb{N}$ such that for any $n>n_{0}$ it holds

$$
\mathbf{E}\left[\left(\xi(t)-\xi_{k, h_{n}}\right)^{2}\right]<\varepsilon
$$

for any F-transform component $\xi_{k, h_{n}}$ w.r.t. $\quad \mathbf{A}_{h_{n}}$ and $t \in \mathbb{R}$ such that $\left|c_{k}-t\right| \leq h_{n}$.

Proof: By analogous arguments as in the proof of previous theorem, for an arbitrary $\mathbf{A}_{h_{n}}$ and $c_{k}$ (a node from $\mathbf{A}_{h_{n}}$ ), we obtain

$$
\begin{gathered}
\mathbf{E}\left[\left(\xi(t)-\xi_{k, h_{n}}\right)^{2}\right]= \\
\frac{1}{h_{n}^{2}} \mathbf{E}\left[\left(\int_{c_{k-1}}^{c_{k+1}}(\xi(s)-\xi(t)) A_{k}(s) d s\right)^{2}\right] \\
\leq \frac{4}{h_{n}} \int_{c_{k-1}-t}^{c_{k+1}-t} B^{*}(\tau) d \tau \leq \frac{4}{h_{n}} \int_{-2 h_{n}}^{2 h_{n}} B^{*}(\tau) d \tau,
\end{gathered}
$$

where $\left|c_{k}-t\right| \leq h_{n}$. A simple consequence of the assumption (18) is

$$
\lim _{h \rightarrow \infty} \frac{4}{h} \int_{-2 h}^{2 h} B^{*}(\tau) d \tau=0 .
$$

Therefore, there exists $n_{0} \in \mathbb{N}$ such that for any $n>n_{0}$ it holds

$$
\frac{4}{h_{n}} \int_{-2 h_{n}}^{2 h_{n}} B^{*}(\tau) d \tau<\varepsilon,
$$

which concludes the proof.

Theorem 7 Let $\xi(t)$ satisfy (18) and $\left(\mathbf{A}_{h_{n}}\right)_{n=1}^{\infty}$ be a sequence of UFPs such that $\lim _{n \rightarrow \infty} h_{n}=0$. Then, for any $\varepsilon>0$, there exists $n_{0} \in \mathbb{N}$ such that for any $n>n_{0}$ it hods

$$
\mathbf{E}\left[\left(\xi(t)-\xi_{k, h_{n}}\right)\left(\xi(t)-\xi_{k+1, h_{n}}\right)\right]<\varepsilon
$$

for any $F$-transform components $\varepsilon_{k, h_{n}}$ and $\varepsilon_{k+1, h_{n}}$ w.r.t. $\quad \mathbf{A}_{h_{n}}$ and $t \in \mathbb{R}$ such that $\left|c_{k}-t_{0}\right| \leq h_{n}$ and $\left|c_{k+1}-t_{0}\right| \leq h_{n}$.

Proof: By the previous theorem, there exists $n_{0}$ such that for any $n>n_{0}$

$$
\begin{array}{r}
\mathbf{E}\left[\left(\xi(t)-\xi_{k, h_{n}}\right)^{2}\right]<\varepsilon, \\
\mathbf{E}\left[\left(\xi(t)-\xi_{k+1, h_{n}}\right)^{2}\right]<\varepsilon
\end{array}
$$

hold for any $t \in \mathbb{R}$ with $\left|c_{k}-t\right| \leq h_{n}$ and $\left|c_{k+1}-t\right| \leq$ $h_{n}$. From (9), we simply obtain

$$
\begin{gathered}
\mathbf{E}\left[\left(\xi(t)-\xi_{k, h_{n}}\right)\left(\xi(t)-\xi_{k+1, h_{n}}\right)\right] \leq \\
\left(\mathbf{E}\left[\left(\xi(t)-\xi_{k, h_{n}}\right)^{2}\right]\right)^{\frac{1}{2}}\left(\mathbf{E}\left[\left(\xi(t)-\xi_{k+1, h_{n}}\right)^{2}\right]\right)^{\frac{1}{2}}<\varepsilon,
\end{gathered}
$$

which concludes the proof.

The last theorem of this section is a version of Theorem 2 in [6] for stationary processes.
Theorem 8 Let $\xi(t)$ satisfy (18) and $\left(\mathbf{A}_{h_{n}}\right)_{n=1}^{\infty}$ be a sequence of UFPs such that $\lim _{n \rightarrow \infty} h_{n}=0$. Then, the corresponding sequence of inverse $F$ transforms

$$
\hat{\xi}_{h_{1}}(t), \hat{\xi}_{h_{2}}(t), \ldots
$$

converges in the mean square to $\xi(t)$, i.e.,

$$
\underset{n \rightarrow \infty}{\lim } \hat{\xi}_{h_{n}}(t)=\xi(t)
$$

for any $t \in \mathbb{R}$.

Proof: Let $\mathbf{A}_{h_{1}}, \mathbf{A}_{h_{2}}, \ldots$ be a sequence of UFPs with $\lim _{n \rightarrow \infty} h_{n}=0$ and let $\varepsilon>0$ be arbitrary. Recall that $\hat{\xi}_{h_{n}}(t)=\sum_{k \in \mathbb{Z}} \xi_{k, h_{n}} A_{k}(t)$ for any $t \in \mathbb{R}$. By Theorems 6 and 7 , there exists $n_{0} \in \mathbb{N}$ such that for any $n>n_{0}$ it holds

$$
\begin{aligned}
\mathbf{E}\left[\left(\xi(t)-\xi_{k, h_{n}}\right)^{2}\right] & <\varepsilon, \\
\mathbf{E}\left[\left(\xi(t)-\xi_{k+1, h_{n}}\right)^{2}\right] & <\varepsilon, \\
\mathbf{E}\left[\left(\xi(t)-\xi_{k, h_{n}}\right)\left(\xi(t)-\xi_{k+1, h_{n}}\right)\right] & <\varepsilon
\end{aligned}
$$

for any $k \in \mathbb{N}$ and $t \in \mathbb{R}$ such that $\left|c_{k}-t\right| \leq h_{n}$ and $\left|c_{k+1}-t\right| \leq h_{n}$. If $t \in \mathbb{R}$ is fixed, then for any $n>n_{0}$ there exists $c_{k}, c_{k+1}$ satisfying the previous inequalities. Moreover, according to the definition of uniform fuzzy partitions, we have

$$
\hat{\xi}_{h_{n}}(t)=\xi_{k, h_{n}} A_{k}(t)+\xi_{k+1, h_{n}} A_{k+1}(t) .
$$

Hence, we obtain

$$
\begin{gathered}
\mathbf{E}\left[\left(\hat{\xi}_{h_{n}}(t)-\xi(t)\right)^{2}\right]= \\
\mathbf{E}\left[\left(\xi_{k, h_{n}} A_{k}(t)+\xi_{k+1, h_{n}} A_{k}(t)-\xi(t)\right)^{2}\right]= \\
\mathbf{E}\left[\left(\left(\xi_{k, h_{n}}-\xi(t)\right) A_{k}(t)+\left(\xi_{k+1, h_{n}}-\xi(t)\right) A_{k+1}(t)\right)^{2}\right]= \\
\mathbf{E}\left[\left(\xi_{k, h_{n}}-\xi(t)\right)^{2}\right]\left(A_{k}(t)\right)^{2}+ \\
+2 \mathbf{E}\left[\left(\xi_{k, h_{n}}-\xi(t)\right)\left(\xi_{k+1, h_{n}}-\xi(t)\right)\right] A_{k}(t) A_{k+1}(t)+ \\
+\mathbf{E}\left[\left(\xi_{k+1, h_{n}}-\xi(t)\right)^{2}\right]\left(A_{k+1}(t)\right)^{2}< \\
\varepsilon\left(\left(A_{k}(t)\right)^{2}+2 A_{k}(t) A_{k+1}(t)+\left(A_{k+1}(t)\right)^{2}\right)= \\
\varepsilon\left(A_{k}(t)+A_{k+1}(t)\right)=\varepsilon .
\end{gathered}
$$

for any $n>n_{0}$, which concludes the proof.

\section{Noise reduction of $\xi(t)$ by F-transform}

Let $\mathbf{A}_{h}=\left(A_{k}\right)_{k \in \mathbb{Z}}$ be a fixed uniform fuzzy partition determined by $\left(K, h, c_{0}\right)$ over which the Ftransform is applied. Let $\left(c_{k}\right)_{k \in \mathbb{Z}}$ denote the respective nodes. Recall that we assume only stationary processes $\xi(t)$ such that the following integral exists for any $k \in \mathbb{Z}$

$$
\xi_{k}=\frac{1}{h} \int_{c_{k-1}}^{c_{k+1}} \xi(t) A_{k}(t) d t
$$

and, moreover,

$$
\mathbf{E}[\xi(t)]=0 \text { and } \operatorname{Var}[\xi(t)]=\sigma^{2}
$$


for any $t \in \mathbb{R}$.

Let us denote

$$
\begin{aligned}
\mathcal{I}_{k l} & =\frac{1}{h^{2}} \int_{c_{k-1}}^{c_{k+1}} \int_{c_{l-1}}^{c_{l+1}}|B(t-s)| A_{k}(t) A_{l}(s) d t d s, \\
\mathcal{I}_{h} & =\frac{1}{h} \int_{-h}^{h} B(\tau) K_{h}(\tau) d \tau,
\end{aligned}
$$

where $K_{h}(t)=K(t / h)$ The following theorem characterizes the mean and variance of the component $\xi_{k}$.

Theorem 9 Let $\xi(t)$ be a stationary random process and $\mathbf{A}_{h}$ be a uniform fuzzy partition. Then, for any $k, l \in \mathbb{Z}$, we have

(i) $\mathbf{E}\left[\xi_{k}\right]=0$,

(ii) $\left|\operatorname{Cov}\left[\xi_{k}, \xi_{l}\right]\right| \leq \mathcal{I}_{k l} \leq \sigma^{2}$,

(iii) if $|B(\tau)|$ is a non-increasing function in $[0, \infty)$, then

$$
\left|\operatorname{Cov}\left[\xi_{k}, \xi_{l}\right]\right| \leq \mathcal{I}_{h} \leq \sigma^{2}
$$

Remark 3 Obviously, the assumption on $B(\tau)$ in (iii) can be equivalently expressed supposing that $B(\tau)$ is a non-negative function that is nonincreasing on $[0, \infty)$. Thus, we assume that the dependence of random variables non-increases for increasing time differences.

Proof: (i) It immediately follows from the equality

$$
\mathbf{E}\left[\int_{c_{k-1}}^{c_{k+1}} R(t) A_{k}(t) d t\right]=\int_{c_{k-1}}^{c_{k+1}} \mathbf{E}[R(t)] A_{k}(t) d t .
$$

(ii) From (ii) of Theorem 1, one can simply show that

$$
\begin{gathered}
\left|\mathbf{E}\left[\xi_{k} \xi_{l}\right]\right| \leq \frac{1}{h^{2}} \int_{c_{k-1}}^{c_{k+1}} \int_{c_{l-1}}^{c_{l+1}}|B(t, s)| A_{k}(t) A_{l}(s) d t d s \\
=\mathcal{I}_{k l} \leq \frac{\sigma^{2}}{h^{2}} \int_{c_{k-1}}^{c_{k+1}} \int_{c_{l-1}}^{c_{l+1}} A_{k}(t) A_{l}(s) d t d s=\sigma^{2} .
\end{gathered}
$$

The statement follows from $\left|\operatorname{Cov}\left[\xi_{k}, \xi_{l}\right]=\right| \mathbf{E}\left[\xi_{k} \xi_{l}\right] \mid$.

(iii) Let $|B(\tau)|$ be a non-increasing function. Put

$$
H_{k l}(t)=\frac{1}{h} \int_{c_{l-1}}^{c_{l+1}}|B(t, s)| A_{l}(s) d s .
$$

for any $t \in\left[c_{k-1}, c_{k+1}\right]$. Using the substitution $\tau=$ $s-c_{l}$, one can rewrite $(25)$ as

$$
H_{k l}(t)=\frac{1}{h} \int_{h}^{-h}\left|B\left(\tau-\left(t-c_{l}\right)\right)\right| K_{h}(\tau) d \tau .
$$

Since $|B(\tau)|$ is a non-negative even function, we obtain that

$$
H_{k l}(t) \leq H_{k l}\left(c_{l}\right)=\mathcal{I}_{h}
$$

Indeed, put $\Delta=t-c_{l}$ and $B^{\prime}(\tau)=B(\tau-\Delta)$. Without loss of generality, suppose that $\Delta \geq 0$. One can simply check that

$$
|B(\tau)|-\left|B^{\prime}(\tau)\right|=\left|B^{\prime}\left(\tau^{\prime}\right)\right|-\left|B\left(\tau^{\prime}\right)\right|
$$

for any $\tau, \tau^{\prime} \in \mathbb{R}$ such that $\tau+\tau^{\prime}=\Delta$. A simple consequence of $\Delta>0$ is

$$
\begin{aligned}
& \left(|B(\tau)|-\left|B^{\prime}(\tau)\right|\right) K_{h}(\tau) \geq \\
& \quad\left(\left|B^{\prime}\left(\tau^{\prime}\right)\right|-\left|B\left(\tau^{\prime}\right)\right|\right) K_{h}\left(\tau^{\prime}\right)
\end{aligned}
$$

for any $\tau, \tau^{\prime} \in \mathbb{R}$ such that $\tau \leq \frac{\Delta}{2}$ and $\tau+\tau^{\prime}=\Delta$. Hence, we obtain

$$
\begin{aligned}
& \int_{-\infty}^{\frac{\Delta}{2}}\left(|B(\tau)|-\left|B^{\prime}(\tau)\right|\right) K_{h}(\tau) d \tau \geq \\
& \int_{\frac{\Delta}{2}}^{+\infty}\left(\left|B^{\prime}(\tau)\right|-|B(\tau)|\right) K_{h}(\tau) d \tau,
\end{aligned}
$$

which implies

$$
\begin{gathered}
\int_{-h}^{h}|B(\tau)| K_{h}(\tau) d \tau=\int_{-\infty}^{+\infty}|B(\tau)| K_{h}(\tau) d \tau \geq \\
\int_{-\infty}^{+\infty}\left|B^{\prime}(\tau)\right| K_{h}(\tau) d \tau=\int_{-h}^{h}\left|B^{\prime}(\tau)\right| K_{h}(\tau) d \tau .
\end{gathered}
$$

Using (ii), we obtain

$$
\begin{gathered}
\left|\operatorname{Cov}\left[\xi_{k}, \xi_{l}\right]\right| \leq \mathcal{I}_{k l}=\frac{1}{h} \int_{c_{k-1}}^{c_{k+1}} H_{k l}(t) A_{k}(t) d t \leq \\
\frac{1}{h} \int_{c_{k-1}}^{c_{k+1}} \mathcal{I}_{h} A_{k}(t) d t=\mathcal{I}_{h} \leq \sigma^{2}
\end{gathered}
$$

which concludes the proof.

Remark 4 One can see that the variability of component of F-transform is a special case of (ii) and (iii) of the previous lemma. Namely, $\operatorname{Var}\left[\xi_{k}\right] \leq \sigma^{2}$ and if $\mathcal{I}_{k k}<\sigma^{2}$ (or $\mathcal{I}_{h}<\sigma^{2}$ under the assumption declared in (iii)).

Remark 5 An interesting consequence of (ii) (or (iii)) of the previous lemma is that for higher values of $h$ the variance of F-transform components decreases. A similar effect can be obtained if the dependencies among random variables in different times are weaker (close to zero) even for times which differences are very small. In other words, if the value of double integral in $\mathcal{I}_{k l}$ (or integral in $\left.\mathcal{I}_{h}\right)$ is close to zero, then $\left|\operatorname{Cov}\left[\xi_{k}, \xi_{l}\right]\right|$ is close to zero.

Now, we will proceed with the analysis of mean and variance of inverse F-transform of $\xi(t)$. In the previous section, we showed that $\hat{\xi}(t)$ can approximate $\xi(t)$ with an arbitrary precision. Therefore, it is natural to expect that the same holds also for functions obtained using the inverse F-transform. The following theorem shows that our conjecture is right which can be also used, for example, for a noise reduction in time series. 
Theorem 10 Let $\hat{\xi}(t)$ denote the inverse $F$ transform of a stationary process $\xi(t)$ over a uniform fuzzy partition $\mathbf{A}_{h}$. Then,

(i) $\mathbf{E}[\hat{\xi}(t)]=\mathbf{E}[\xi(t)]=0$,

(ii) $\operatorname{Var}[\hat{\xi}(t)] \leq \sum_{i, j=0}^{1} \mathcal{I}_{k+i, k+j} A_{k+i}(t) A_{k+j}(t) \leq$ $\sigma^{2}$

(iii) if $|B(\tau)|$ is a non-increasing function in $[0, \infty)$, then $\operatorname{Var}[\hat{\xi}(t)] \leq \mathcal{I}_{h} \leq \sigma^{2}$,

holds for any $t \in \mathbb{R}$.

Proof: (i) It immediately follows from (i) of the previous theorem and the equality (supposing that $\left.t \in\left[c_{k}, c_{k-1}\right]\right)$

$$
\hat{\xi}(t)=\xi_{k} A_{k}(t)+\xi_{k+1} A_{k+1}(t) .
$$

(ii) Using (ii) of Theorem 9, we obtain

$$
\begin{gathered}
\operatorname{Var}[\hat{\xi}(t)] \leq \sum_{i=0}^{1} \sum_{j=0}^{1}\left|\mathbf{C o v}\left[\xi_{k}, \xi_{l}\right]\right| A_{k+i}(t) A_{k+j}(t) \leq \\
\sum_{i=0}^{1} \sum_{j=0}^{1} \mathcal{I}_{k+i, k+j} A_{k+i}(t) A_{k+j}(t) \leq \sigma^{2} .
\end{gathered}
$$

(iii) It can be proved similarly to the previous statement using (iii) of Theorem 9.

Remark 6 Similarly to Remark 5, we can deduce that a higher reduction of variability in filtered out stationary process $\hat{\xi}(t)$ is reached for higher values of $h$ and lower values of double integral in $\mathcal{I}_{k l}$ or (integral in $\mathcal{I}_{h}$ ).

\section{Conclusion}

In this paper, we analyzed the (weakly) stationary processes. We showed that under certain assumptions the fuzzy transform can be used for an approximation of this type of stationary process as well as for a reduction of variability. Although, our results are only preliminary ones and they are far from the known results on the discrete representation of stochastic processes (see, e.g., [12]), we believe that they support and give us rational arguments to continue in the investigation of stationary processes in the context of the F-transform.

\section{Acknowledgment}

The authors would like to thank to Department of the Navy Grant N62909-12-1-7039 issued by Office of Naval Research Global. The United States Government has a royalty-free license throughout the world in all copyrightable material contained herein. Additional support was given also by the European Regional Development Fund in the IT4Innovations Centre of Excellence project (CZ.1.05/1.1.00/02.0070)

\section{References}

[1] V. Novák, I. Perfilieva, and V. Kreinovich. Ftransform in the analysis of periodic signals. In M. Inuiguchi, Y. Kusunoki, and M. Seki, editors, Proc. $15^{\text {th }}$ Czech-Japan Seminar on Data Analysis and Decision Making under Uncertainty, Osaka, Japan, 2012. Osaka University.

[2] V. Novák, I. Perfilieva, M. Holčapek, and V. Kreinovich. Filtering out high frequencies using f-transform. Submitted to Fuzzy Sets and Systems.

[3] A.M. Yaglom. An introduction to the theory of stationary random functions. Revised English ed. Translated and edited by Richard A. Silverman. Englewood Cliffs, NJ: Prentice-Hall, Inc. XIII, 235 p. , 1962.

[4] P.J. Brockwell and R.A. Davis. Introduction to Time Series and Forecasting. Springer-Verlag New York, 2002.

[5] A.H. Jazwinski. Stochastic processes and filtering theory. Mineola, NY: Dover Publications. xiv, 376 p. , 2007.

[6] I. Perfilieva. Fuzzy transforms: Theory and applications. Fuzzy sets syst., 157(8):993-1023, 2006.

[7] I. Perfilieva and M. Daňková. Towards ftransform of a higher degree. In Proceedings of IFSA/EUSFLAT 2009, pages 585-588, Lisbon, Portugal, 2009.

[8] I. Perfilieva. Fuzzy transforms: A challenge to conventional transforms. In P.W. Hawkes, editor, Advances in Images and Electron Physics, 147, pages 137-196. Elsevier Academic Press, San Diego, 2007.

[9] I. Perfilieva, M. Daňková, and B. Bede. Towards a higher degree $F$-transform. Fuzzy Sets Syst., 180(1):3-19, 2011.

[10] M. Holčapek and T. Tichý. A smoothing filter based on fuzzy transform. Fuzzy Sets Syst., 180(1):69-97, 2011.

[11] K. Loquin and O. Strauss. Histogram density estimators based upon a fuzzy partition. Stat. Probab. Lett., 78(13):1863-1868, 2008.

[12] K. L. Jordan. Discrete representation of random signals. Technical Report 378, Massachusetts Institute of Technology Research Laboratory of Electronics, 1961. 